\title{
Correction to: A Prospective Investigation of the Impact of Distinct Posttraumatic (PTSD) Symptom Clusters on Suicidal Ideation
}

\author{
Maria Panagioti ${ }^{1} \cdot$ Ioannis Angelakis $^{2} \cdot$ Nicholas Tarrier $^{3} \cdot$ Patricia Gooding $^{4}$
}

Published online: 4 January 2021

(c) Springer Science+Business Media, LLC, part of Springer Nature 2021

\section{Correction to: \\ Cognitive Therapy and Research (2017) 41:645-653 https://doi.org/10.1007/s10608-016-9829-2}

The original version of this article contained a mistake in the Table 3. In Table 3: Two rows were accidentally cut out which reported the B coefficients and standard errors for two variables in the multiple regression model, the hyperoursal symptom cluster, avoidance/numbing symptom cluster. The corrected Table 3 is presented below.

The original article can be found online at https://doi.org/10.1007/ s10608-016-9829-2.

Maria Panagioti maria.panagioti@manchester.ac.uk

1 Division of Population Health, Health Services Research \& Primary Care, University of Manchester, Williamson Building, Oxford Road, Manchester M13 9PL, UK

2 School of Psychology, University of South Wales, Pontypridd, UK

3 Institute of Psychiatry, Kings College London, London, UK

4 School of Psychological Sciences, University of Manchester, Manchester, UK 
Table 3 Results from the regression analyses examining the three PTSD symptom clusters at baseline as predictors of suicidal ideation at follow-up

\begin{tabular}{llllll}
\hline Step & Variable entered & B & SE & Total $\mathrm{R}^{2}\left(f^{2}\right)$ & $\Delta \mathrm{R}^{2}\left(f^{2}\right)$ \\
\hline 1 & Baseline suicidal ideation & $0.504^{* * *}$ & 0.023 & $0.75^{* * *}(0.30)$ & \\
& Severity of depressive symptoms & $0.021^{* *}$ & 0.010 & & \\
& Defeat/entrapment & $0.044^{* *}$ & 0.012 & & \\
& Baseline suicidal ideation & $0.422^{* * *}$ & 0.109 & $0.82 * *(0.46)$ & $0.07 * *(0.08)$ \\
& Severity of depressive symptoms & $0.022^{*}$ & 0.011 & & \\
Defeat/entrapment & $0.028^{* *}$ & 0.011 & & \\
Re-experiencing symptom cluster & 0.008 & 0.020 & & \\
Avoidance/Numbing symptom cluster & -0.010 & 0.018 & & \\
Hyperarousal symptom cluster & $0.100^{* *}$ & 0.018 & &
\end{tabular}

${ }^{*} p<0.05 ; * * p<0.01 ; * * * p<0.001$.

Publisher's Note Springer Nature remains neutral with regard to jurisdictional claims in published maps and institutional affiliations. 